\section{Prevalência e intensidade de infecção por Ascaridia sp. em papagaios-verdadeiros}

\author{
Prevalence and intensity of \\ Ascaridia sp. infection on \\ turquoise-fronted parrot
}

Universidade Federal Rural do Rio de Janeiro (UFRRJ),

Seropédica, RJ, Brasil

* Correspondência: roberta.jordano@gmail.com

Submetido: 5 out 2020 | Aprovado: 8 out 2021

DOI: http://dx.doi.org/10.7213/acad.2021.19011

Rev. Acad. Ciênc. Anim. 2021;19:e19011

\section{Resumo}

Amazona aestiva é uma espécie com grande representatividade no Centro de Triagem de Animais Silvestres do Rio de Janeiro (CETAS-RJ). Infecções parasitárias ainda são um dos principais problemas de saúde das aves silvestres mantidas em cativeiro. Objetivou-se neste estudo descrever a prevalência e intensidade de infecção por Ascaridia sp. em carcaças de papagaios-verdadeiros (Amazona aestiva Linnaeus, 1758) oriundos do CETAS-RJ. Dos animais submetidos à necropsia, 48\% (12/25) estavam infectados. No intestino de um indivíduo foram encontrados 172 parasitos. Os parasitos encontrados foram identificados pelo setor de parasitologia da Universidade Federal Rural do Rio de Janeiro (UFRRJ). Todos os animais afetados (12/12) albergavam endoparasitas do gênero Ascaridia sp. no intestino e em uma das carcaças (1/12) também foram

\author{
Roberta Cristine Jordano Brito (1) \\ Andressa Kagohara (D) \\ Luciano da Silva Alonso (D) \\ Luiz Ricaro de Oliveira Roberto (D) \\ Thaís Ribeiro Correia Azevedo (i) \\ Lauren de Freitas Baqueiro (D) \\ Daniel de Almeida Balthazar (D)
}

observados dois indivíduos do mesmo parasito no inglúvio. De acordo com resultados obtidos, concluise que as carcaças de Amazona aestiva analisadas estavam com alta infecção parasitária. Limpeza do local, quarentena das aves novas no plantel e medicação às aves positivas são medidas de controle que devem ser instituídas, visando quebrar o ciclo de transmissão.

Palavras-chave: Ascaridíase. Psitaciforme. Superlotação.

\section{Abstract}

Amazona aestiva is a species with great representativeness at the Centro de Triagem de Animais Silvestres (CETAS-RJ). Parasitic infections are some of the primary health problems found in wild birds that are kept in captivity. The objective of this study was to describe the prevalence and intensity of Ascaridia sp. on carcasses of turquoise-fronted parrot (Amazona aestiva Linnaeus, 1758) from CETAS-RJ. Of the animals subjected to necropsy, 48\% (12/25) were infected. In the intestines of one individual were found 172 parasites. The parasites found were identified by the department of parasite studies of the Universidade Federal Rural do Rio de Janeiro (UFRRJ). All the affected individuals (12/12) were positive for Ascaridia sp. in the intestines, and in one of the carcasses (1/12) were also observed two individuals 
of this parasite in the ingluvies. According to the results obtained, it can be concluded that the carcasses of de Amazona aestiva analysed had a high level of parasite infection. Cleaning the facilities, quarantine of new birds, and medication to treat the positive birds are control measures that must be instituted aiming to break the transmission cycle.

Keywords: Ascaridiasis. Psittaciform. Overcrowded.

\section{Introdução}

Infecções parasitárias são um dos principais problemas de saúde das aves silvestres mantidas sob cuidados humanos (Greiner e Ritchie, 1994; GómezPuerta et al., 2008; Oliveira et al., 2018). Existe uma variedade de helmintos que parasitam aves, sendo os principais grupos representantes os nematoides e cestoides (Rennó et al., 2008).

Os endoparasitas podem causar infecções e doenças conforme o tipo de manejo, resistência dos animais, potencial biótico dos patógenos e a própria rusticidade das aves (Snak et al., 2014). Além disso, de acordo com Melo et al. (2013), os danos ao hospedeiro e os sintomas apresentados dependem da patogenicidade e intensidade da infecção, além do estado geral do animal. Mesmo um parasito de baixa patogenicidade pode causar doença clínica grave se concomitante com imunossupressão, estresse ou outras doenças. Um importante agravante é a grande quantidade de parasitos que, ao morrerem, após o protocolo de desparasitação do hospedeiro, liberam neurotoxinas que podem causar convulsões, incoordenação motora e, por fim, a morte (Rennó et al., 2008). Em alguns casos também pode ocorrer obstrução intestinal e intussuscepção devido à alta carga parasitária de Ascaridia sp. (Melo et al., 2013).

Estudos descrevem o gênero Ascaridia sp. destacando a sua presença especificamente em psitacídeos (Rennó et al., 2008; Costa et al., 2010; Snak et al., 2014). O ciclo de vida do parasito é direto e ocorre pela ingestão de ovos embrionados presentes no ambiente. Após a sua ingestão, os ovos eclodem no trato gastrointestinal, onde os parasitos se desenvolvem e se reproduzem. As fêmeas gravídicas liberam novos ovos, não embrionados, que são eliminados nas fezes. No ambiente, entre 5 e 14 dias e em condições favoráveis de temperatura $\left(22^{\circ} \mathrm{C}-34^{\circ} \mathrm{C}\right)$ e umidade, eles tornam-se embriões e, dessa forma, infectantes (Fedynich, 2008). Há relatos de algumas espécies que utilizam anelideos como hospedeiros paratênicos (Fedynich, 2008). Após o embrionamento, os ovos podem permanecer viáveis no ambiente durante meses, mesmo quando expostos a temperaturas adversas $\left(-12^{\circ} \mathrm{C}-57^{\circ} \mathrm{C}\right)$ (Ackert, 1931; Christenson et al., 1942). Sendo assim, o ponto crítico para controle deste parasito é impedir o embrionamento do ovo, mantendo o ambiente seco e higienizando fômites como bebedouros, comedouros e poleiros rotineiramente. Recomendase também manter as aves em viveiros suspensos, impedindo seu contato direto com as fezes e, consequentemente, a perpetuação do ciclo de transmissão (Mosgovoi, 1953; Levine, 1968; Greiner e Ritchie, 1994). Além disso, após confirmação de parasitismo, o uso de anti-helmínticos também é recomendado (Fedynich, 2008).

Dada a importância do papagaio-verdadeiro (Amazona aestiva Linnaeus, 1758) no quantitativo de aves vítimas de comércio ilegal, sua participação como animal de companhia e a representatividade nos Centros de Triagem de Animais Silvestres (CETAS), a identificação de parasitos encontrados em necropsia contribui com novas abordagens ecológicas para a proteção da espécie. Segundo Souza et al. (2018), o estudo das infecções parasitárias e da interação parasito-hospedeiro são ferramentas de auxílio nos programas de conservação e preservação de espécies, pois os animais silvestres são considerados hospedeiros e reservatórios, podendo inferir na saúde dos ecossistemas e ambientes naturais e domésticos (Freitas et al., 2002). Além disso, sendo o CETAS um importante centro de recuperação de animais que podem retornar à natureza, entender a dinâmica das doenças presentes no plantel é importante para a elaboração de estratégias preventivas e para impedir que haja a introdução de novos agentes na natureza, o que pode causar prejuízos imprevisíveis para as espécies selvagens (Prenter et al., 2004).

Objetivou-se neste estudo descrever a prevalência e intensidade de infecção por Ascaridia sp. em carcaças de papagaios-verdadeiros. A identificação de parasitos em Amazona aestiva é um indicador de saúde populacional útil em programas de manejo e preservação da espécie. 


\section{Material e métodos}

Para a realização deste trabalho foram utilizados 25 cadáveres de papagaios-verdadeiros provenientes do CETAS-RJ, localizado no município de Seropédica $\left(22^{\circ} 44^{\prime} \mathrm{S}, 43^{\circ} 42^{\prime} \mathrm{W}\right)$. A cessão das carcaças pelo CETAS-RJ foi realizada conforme Instrução Normativa $n^{\circ} 179$ (Brasil, 2008) e os trabalhos desenvolvidos mediante autorização para atividades com finalidade científica (SISBIO nº 65486-1).

As necropsias foram realizadas por três pessoas habilitadas, as quais atenderam preceitos normativos e uso de equipamentos de proteção individual adequados para a atividade, como luvas, jalecos e sapatos fechados. Para a abertura

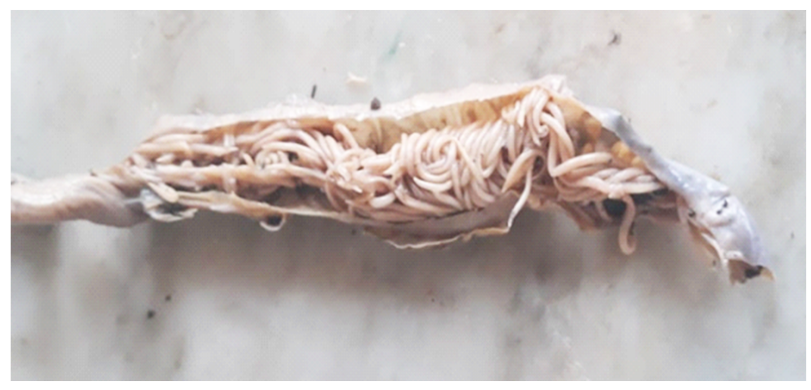

Figura 1 - Parasitos em intestino da primeira ave (Amazona aestiva) necropsiada (Seropédica, 2019).

\section{Análise estatística}

Em técnicas gerais para a coleta e preparação de helmintos de aves, Amato e Amato (2010) definem as variáveis em que foram baseados os resultados do presente estudo:

- Prevalência: número de hospedeiros infectados dividido pelo número de hospedeiros examinados. Resultado expresso em percentagem.

- Intensidade de infecção: número de indivíduos pertencentes a uma espécie de parasito encontrado em um hospedeiro infectado/infestado.

- Intensidade média: número total de parasitos de uma determinada espécie encontrado em uma amostra dividido pelo número total de hospedeiros infectados com aquela espécie de parasito.

- Abundância média: número total de uma determinada espécie de parasito em uma amostra/ número total de hospedeiros. das carcaças foram utilizadas tesouras, bisturi e pinças na abertura da cavidade celomática. Inicialmente, os animais foram identificados. Depois, as penas da superfície ventral foram retiradas e, em seguida, os músculos peitoral, abdominal e o osso esterno foram removidos, possibilitando a visualização das vísceras. As mesmas foram retiradas e analisadas em busca de possíveis alterações. Logo após a remoção das vísceras, procedeu-se a abertura do trato gastrointestinal e coleta dos parasitos (Figuras 1 e 2), conforme Amato e Amato (2010). No laboratório de parasitologia da Universidade Federal Rural do Rio de Janeiro (UFRRJ), os helmintos foram clarificados em lactofenol de Amann (Amato e Amato 2010) e identificados.

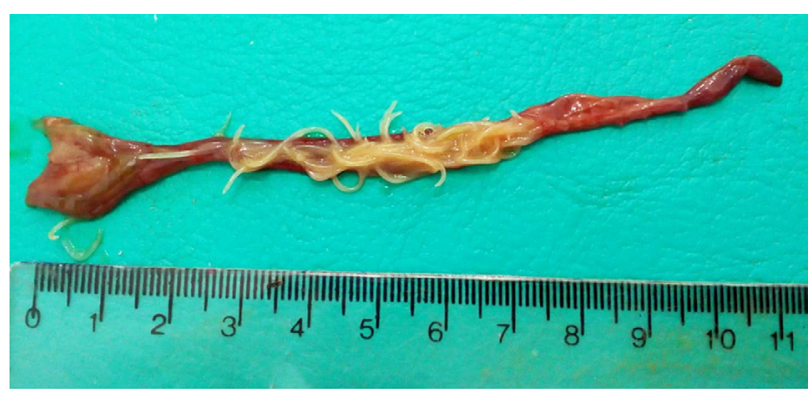

Figura 2 - Parasitos em intestino de ave fresca (Amazona aestiva) não formolizada. (Seropédica, 2019).

- Infrapopulação: é formada por todos os parasitos pertencentes à mesma espécie e encontrados no mesmo local de infecção/infestação de um mesmo hospedeiro, em um determinado momento de tempo.

\section{Resultados e discussão}

Nematódeos são vermes cilíndricos, possuem sistema digestivo completo, dimorfismo sexual e apresentam um formato consistente e organizado. Para sua identificação, observam-se a cavidade corporal e o aparelho digestório (Avelar, 2014). Possuem órgãos tubulares, cavidade pseudocelomática e parede corpórea composta por uma cutícula (variável na espessura), que é responsável pela "proteção" da larva no ambiente (Monteiro et al., 2011). O gênero Ascaridia é um nematoide 
pertencente à família Ascaridiidae e tem como hospedeiros galinha, peru, pato, faisão e outras aves, parasitando o intestino delgado. Segundo Vicente et al. (1995), Ascaridia sp. já foram identificados em 54 espécies de psitacídeos.

O ciclo evolutivo é direto: a ave ingere o ovo contendo L3 (estádio infectante), que eclode no intestino delgado, onde passa a $L 4$ e a adultos (machos e fêmeas); há cópula e a fêmea faz postura dos ovos, que são levados ao meio ambiente pelas fezes. O diagnóstico, feito através de métodos de pesquisa de ovos, é mais viável e mais preciso através de necropsia parasitológica.

Dos animais submetidos à necropsia no presente estudo, 48\% (12/25) se apresentaram parasitados no interior do trato gastrointestinal (Tabela 1). Nestes, foram encontrados 649 parasitos, sendo todas as infrapopulações identificadas pertencentes ao gênero Ascaridia sp. O maior número observado em um único indivíduo foi de 172 parasitos no intestino delgado (Figura 1). O menor número encontrado foi de um parasito, no mesmo local. Além disso, também foram observados dois em inglúvio, sendo que a presença de parasitos neste último pode ocorrer devido à eclosão dos ovos ingeridos no trato gastrointestinal superior (Ackert, 1931; Ruff, 1984).

A análise estatística revelou prevalência de infecções pelo gênero Ascaridia sp. (48\%), com abundância média de 25,96\% e intensidade de infecção média de 54,08\%. A idade dos animais avaliados não foi informada e o sexo não foi identificado durante o exame necroscópico.

Tabela 1 - Intensidade de infecção por Ascaridia spp. em carcaças de Amazona aestiva oriundas do CETAS-RJ

\begin{tabular}{ccccc}
\hline $\begin{array}{c}\text { Número de } \\
\text { hospedeiros }\end{array}$ & $\begin{array}{c}\text { Hospedeiro } \\
\text { (Ordem de necropsia) }\end{array}$ & $\begin{array}{c}\text { Sítio de infecção } \\
\text { (Local) }\end{array}$ & $\begin{array}{c}\text { Intensidade de } \\
\text { infecção } \\
\text { (nº de parasitos) }\end{array}$ & $\begin{array}{c}\text { Infrapopulação } \\
\text { (Espécie de } \\
\text { parasito) }\end{array}$ \\
\hline 1 & Animal 1 & Intestino delgado & 172 & Gênero: Ascaridia \\
2 & Animal 3 & Intestino delgado & 1 & Gênero: Ascaridia \\
3 & Animal 5 & Intestino delgado & 64 & Gênero: Ascaridia \\
4 & Animal 9 & Intestino delgado & 20 & Gênero: Ascaridia \\
5 & Animal 11 & Intestino delgado & 80 & Gênero: Ascaridia \\
6 & Animal 12 & Intestino delgado & 102 & Gênero: Ascaridia \\
7 & Animal 13 & Intestino delgado & 6 & Gênero: Ascaridia \\
8 & Animal 16 & Intestino delgado & 35 & Gênero: Ascaridia \\
9 & Animal 19 & Intestino delgado & 53 & Gênero: Ascaridia \\
10 & Animal 21 & Intestino delgado & 67 & Gênero: Ascaridia \\
11 & Animal 23 & Intestino delgado & 48 & Gênero: Ascaridia \\
12 & Animal 25 & Intestino delgado & &
\end{tabular}

A alta infecção parasitária por Ascaridia sp. é comum em aves e é descrita em espécies de psitacideos (Greiner e Ritchie, 1994; Doneley, 2016; Siqueira et al., 2017), sendo que fatores ambientais e intrínsecos, como a superlotação de animais no CETAS e o estresse, podem favorecer a disseminação e reinfecção dos indivíduos parasitados. Visto que a eliminação de ovos é pelas fezes e o ciclo de vida do parasito é direto, feco-oral (Fedynich, 2008; Siqueira et al., 2017), quanto mais animais juntos em um mesmo ambiente, mais fezes e maior a contaminação do mesmo, perpetuando este ciclo. Como estratégia de controle ambiental, devido à resistência dos ovos no ambiente e a desinfetantes, recomenda-se que o ambiente seja mantido seco e que as aves fiquem longe do chão e das fezes. Além disso, o tratamento medicamentoso dos animais positivos deve ser implementado a fim de interromper o ciclo de transmissão (Greiner e Ritchie, 1994; Fedynich, 2008). Diversos anti-helmínticos podem ser utilizados, no entanto, é importante utilizá-los de modo consciente e rotacional para evitar o desenvolvimento de resistência parasitária, que vem crescendo nos últimos anos (Doneley, 2016). 
Os históricos referentes a períodos anteriores à chegada dos indivíduos no CETAS-RJ são variados, desde aves domiciliadas de forma ilegal, resgate de locais de risco, acidentes em rodovias e, em sua maioria, apreensão de tráfico. Sendo que estes fatores podem estar correlacionados com a endoparasitose dos animais (Fedynich, 2008), a adoção de quarentena para animais recém-chegados é uma medida eficaz para auxiliar no controle da disseminação de doenças, incluindo as parasitárias no plantel (Silva e Felippe, 2014). Durante este período é possível realizar exames complementares, como o coproparasitológico, a fim de detectar a presença de parasitos e comorbidades e tratá-los antes que as aves sejam liberadas para o ambiente comum.

\section{Conclusão}

Conclui-se com esse trabalho que a prevalência e intensidade de infecção das aves necropsiadas estão relacionadas com a superpopulação de papagaios-verdadeiros no CETAS-RJ, resultado do grande número de apreensões. Sugere-se redimensionamento e suspensão das instalações para evitar condições ambientais que favoreçam a conclusão do ciclo do parasita, além da adoção de quarentena aos animais recém-chegados e tratamento medicamentoso aos animais positivos a fim de evitar a reinfecção dos animais já presentes no recinto.

\section{Referências}

Ackert JE. The morphology and life history of the fowl nematode Ascaridia lineata (Schneider). Parasitology. 1931; 23(3): 360-79.

Amato JFR, Amato SB. Técnicas gerais para coleta e preparação de helmintos endoparasitas de aves. In: Von Matter S et al. Ornitologia e conservação: ciência aplicada, técnicas de pesquisa elevantamento. Rio de Janeiro: Technical Books; 2010. p. 369-93.

Avelar 1O. Identificação parasitológica e histopatológica das endoparasitose em animais selvagens [dissertação]. Belo Horizonte: Universidade Federal de Minas Gerais; 2014.

Brasil. Instrução Normativa IBAMA n 179, de 25 de junho de 2008. Brasília: Diário oficial da União; 26 jun 2008.
Christenson RO, Earle Jr HH, Butler Jr RL, Creel HH. Studies on the eggs of Ascaridia galli and Heterakis gallinae. Trans Am Microsc Soc. 1942;61(2):191-205.

Costa IA, Coelho CD, Bueno C, Ferreira I, Freire RB. Ocorrência de parasitos gastrintestinais em aves silvestres no município de Seropédica, Rio de janeiro, Brasil. Ciência Animal Brasileira, 2010;11(4) :914-22.

Doneley B. Avian medicime and surgery in practice companion and aviary birds. 2 ed. Boca Raton, FL: CRC Press; 2016. p. 239-40.

Fedynich AM. Heterakis and Ascaridia. In: Atkinson CT, Thomas NJ, Hunter BD. Parasitic disease of wild birds. Ames, lowa: Willey-Blackwell; 2008 p.388-412.

Freitas MFL, Oliveira JB, Cavalcanti MDB, Leite AS, Magalhães VS, Oliveira RA, et al. Parásitos gastrointestinales aves silvestres en cautiverio en el estado de Pernambuco, Brasil. Parasitol Latinoam. 2002;57:50-4.

Gómez-Puerta LA, López-Urbina MT, Gozález AE. Ocurrencia de Ascaridia hermafrodita (Nematode: Ascaridiidae) em el loro de Cabeza Azúl (Pionus menstruus) em Perú. Rev Peru Biol. 2008;15(2):133-5.

Greiner EC, Ritchie BW. Parasites. In: Ritchie BW, Harrison GJ, Harrison LR. Avian medicine: principles and application. Lake Worth, FL: Wingers Publishing; 1994. p. 1007-29.

Levine ND. Nematode parasites of domestic animals and of man. Minneapolis, MN: Burgess Publishing Company; 1968.

Melo CMF, Oliveira JB, Feitosa TF, Vilela VLR, Athayde ACR, Dantas AFM, et al. Parasites of Psittaciformes and Accipitriformes in Paraíba state, Northeastern Brazil. Rev Bras Parasitol Vet. 2013;22(2):314-7.

Monteiro SG. Parasitologia na medicina veterinária. São Paulo: Roca; 2011.

Oliveira LD, Costa LL, Cardoso EMO, Azevedo TM. A criação em cativeiro doméstico de aves silvestres: percepção de moradores do município de Curral Velho - PB. Anais III CONAPESC; 20-22 jun 2018; Campina Grande, PB.

Prenter J, Macneil C, Dick JTA, Dunn AM. Roles of parasites in animal invasions. Trends Ecol Evol. 2004;19(7):385-90. 
Rennó PP, Queiroz FM, Garcia BP, Prado RNA, Simões MM, Souza JPF, et al. Endoparasitose em aves - Revisão de Literatura. R Cient Eletr Med Vet. 2008;(11).

Ruff MD. Nematodes and acanthocephalans. In: Hofstad MS et al. Diseases of poultry. 8 ed. Ames, IA: lowa State University Press; 1984. p. 614-48.

Silva JCR, Felippe PAN. Biossegurança. In: Cubas ZS et al. Tratado de animais selvagens. São Paulo: Roca; 2014. p. 2367-23

Siqueira RAS, Neto TSO, Guerra RR, Vilela VLR, Lucena RB. Clínical, pathological and parasitological aspects of ascaridiasis in Blue-fronted parrot (Amazona estiva) from ilegal wildlife trade in Northeastern Brazil. Braz J Vet Pathol. 2017;10(2):43-6.
Snak A, Lenzi PF, Agostini KM, Delgado LE, Montanucci CR, Zabott MV. Análises coproparasitológicas de aves silvestres cativas. Cienc Anim Bras. 2014;15(4):502-7.

Souza JG, Falcão BMR, Batista LN, Carreiro AN, Medeiros GX, Menezes DJA. Descrição e morfometria do osso quadrado de Amazona aestiva e Diopsitataca nobilis. Cienc Anim. 2018;28(3): 19-22.

Vicente JJ, Rodrigues HO, Gomes DC, Pinto RM. Nematoides do Brasil. Parte IV: nematoides de aves. Rev Bras Zool. 1995;12 (Suppl 1):1-273. 